\title{
Significant Benefits of Afatinib and Apatinib in a Refractory Advanced NSCLC Patient Resistant to Osimertinib: A Case Report
}

\author{
Yuanyang Zhao (D) ${ }^{1, *}$ \\ Yuxing Chen $\mathbb{D}^{1, *}$ \\ Huaying Huang $\mathbb{D}^{1}$ \\ $\mathrm{Xi} \mathrm{\textrm {Li } ^ { 2 }}$ \\ Lin Shao ${ }^{2}$ \\ Hao Ding (ID \\ 'Department of Respiratory Disease, \\ Affiliated People's Hospital of Jiangsu \\ University, Zhenjiang, 212002, People's \\ Republic of China; ${ }^{2}$ Department of \\ Medicine, Burning Rock Biotech, \\ Guangzhou, 510300, People's Republic of \\ China
}

*These authors contributed equally to this work

\begin{abstract}
EGFR-tyrosine kinase inhibitors (TKIs) have revolutionized the treatment for NSCLC. However, acquired drug resistance often occurs after treatment with EGFR-TKIs. EGFR T790M and C797S mutations are the most common resistance mechanism in patients who failed from first- and third- generation EGFR TKI treatments, respectively. However, there is no standard of care for NSCLC harboring EGFR T790M and C797S in-cis. The present case reports a 69 -year-old Chinese man with advanced NSCLC harboring EGFR exon 19-deletion, T790M, cis-C797S, and HER2 amplification who was heavily pre-treated. The patient was then given a combination of afatinib and apatinib and achieved a PFS of more than 10 months. This case showed that afatinib plus apatinib may be a promising therapy for patients with EGFR 19Del-T790M-cis-C797S mutant and HER2 amplified NSCLC
\end{abstract}

Keywords: NSCLC, EGFR-mutant, TKI, afatinib, apatinib

\section{Introduction}

Lung cancer remains the leading cause of cancer-related death worldwide. ${ }^{1}$ Epidermal growth factor receptor $(E G F R)$-tyrosine kinase inhibitors (TKI) have become the first-line choice for treating patients with advanced non-small-cell lung cancer (NSCLC) harboring EGFR activating mutations. Unfortunately, most of these patients inevitably experience acquired resistance within less than one year. ${ }^{2}$ The most common acquired mutation EGFR C797S, which occurs in exon 20, mediates the resistance to second-line osimertinib treatment in 10-26\% of cases. However, there is no further clear-cut therapeutic option targeting this mutation to date.

Here, we report a heavily pre-treated metastatic NSCLC patient with EGFR 19Del-T790M-cis-C797S and HER2 amplification, who benefited from a combination therapy of afatinib and apatinib.

\section{Case Presentation}

A 69-year-old man, a never-smoker, presented to our clinic with back and lower limb pain in January 2013. The treatment history of the patient is summarized in Figure 1. Positron emission tomography/computed tomography (PET/CT) scan showed a left-lobe lung lesion with mediastinal lymphadenopathy and multiple bone metastasis (Figure 2A). Through a transbronchoscopic lung biopsy, he was
Correspondence: Hao Ding

Department of Respiratory Disease, Affiliated People's Hospital of Jiangsu

University, No. 8 Dianli Road, Zhenjiang, Jiangsu, 2 I 2002, People's Republic of China

Tel +86-5I I-889I5360

Email dinghao5715@I63.com 


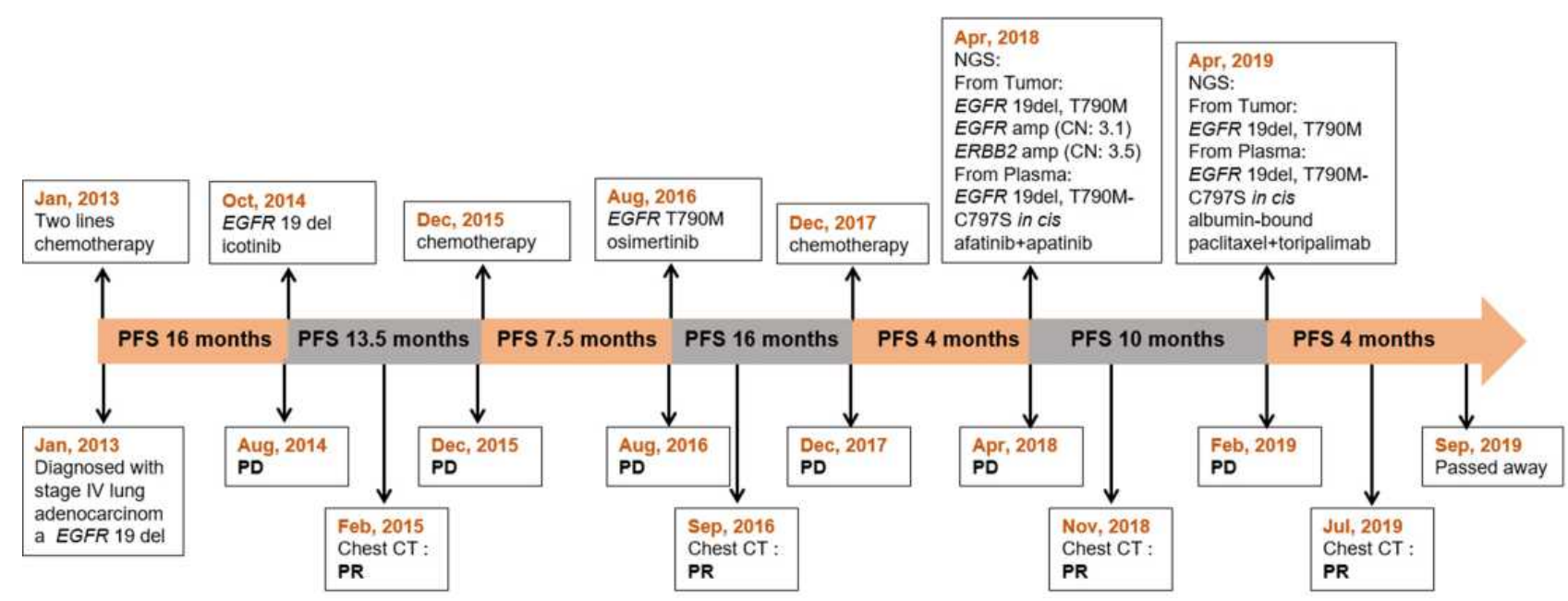

Figure I Diagram of the course of disease management.

diagnosed with lung adenocarcinoma at stage IV. The patient was initially treated with chemotherapy with pemetrexed and cisplatin in the first line and paclitaxel liposome (a dosage form of paclitaxel which is different from nabpaclitaxel) and carboplatin in the second line. He achieved progression-free survival (PFS) of 16 months in total. In August 2014, the patient experienced progressive disease (PD) (Figure 2B). The patient did not have any tissue or liquid biopsy when progressed and his primary lung tissue was subjected to an EGFR alteration test which showed a deletion in EGFR exon 19. He was subsequently administered with icotinib as third-line treatment in October 2014. Icotinib is an EGFR-TKI and had been approved for treating EGFR-mutant NSCLC by National Medical Products Administration (NMPA) in China in 2011. He achieved partial response (PR) based on Response Evaluation Criteria in Solid Tumors 1.1 (RECIST 1.1) with a PFS of 13.5 months (Figure 2C and D). Then he was switched to gemcitabine and nedaplatin for 4 cycles and achieved SD with a PFS of 7.5 months. On August 1, 2016, the disease progressed again and genetic testing indicated EGFR T790M was positive. The patient was subsequently treated with Osimertinib. After 1 month, the patient achieved PR (Figure 2E and F) and lasted for 16 months. After progression, he was switched to vinorelbine in combination with lobaplatin and experienced PD 2.5 months later (Figure 2G).

Subsequently, plasma and biopsy tissue samples of the patient were subjected to capture-based targeted sequencing using a panel consisting of 168 cancer-related genes (Burning Rock Biotech, Guangzhou, China), which revealed EGFR 19 del and EGFR T790M in both plasma and tissue samples. In addition, EGFR C797S in cis with the $E G F R$ T790M was only detected from plasma, while HER2 (ERBB2) amplification with a copy number of 3.5 was only observed in the tissue sample (Figure 3). In April 2018, afatinib combined with apatinib was administered and the patient achieved PR (Figure 2H) with a PFS of more than 10 months.

In February 2019, the patient experienced PD with an enlargement of the left lobe mass (Figure 2I). The fiberoptic bronchoscopy biopsy and molecular testing were performed again. The result showed the retention of EGFR 19 del and T790M without HER2 amplification in tumor tissue, and EGFR 19Del-T790M-cis-C797S in plasma. On April 1, 2019, the treatment regimen was switched to albumin-bound paclitaxel and PD1 inhibitor toripalimab for 4 cycles. During the treatment, the patient had no severe adverse effects. After 2.5 months of treatment, the patient achieved PR (Figure 2J). However, because of the economical difficulty, the treatment was terminated. The patient passed away in September 2019 with an OS (overall survival) of 80 months.

\section{Discussion}

Despite the success of osimertinib both in the first-line and second-line treatment settings in the presence of the EGFR T790M mutation, acquired resistance inevitably occurs and thus limits a prolonged clinical benefit achieved with this drug. The most common acquired tertiary EGFR mutation is EGFR C797S. Importantly, the allelic context in which $\mathrm{C} 797 \mathrm{~S}$ is acquired has potential implications for treatment. It is known that the patients with EGFR T790M and cis-C797S are resistant to all 

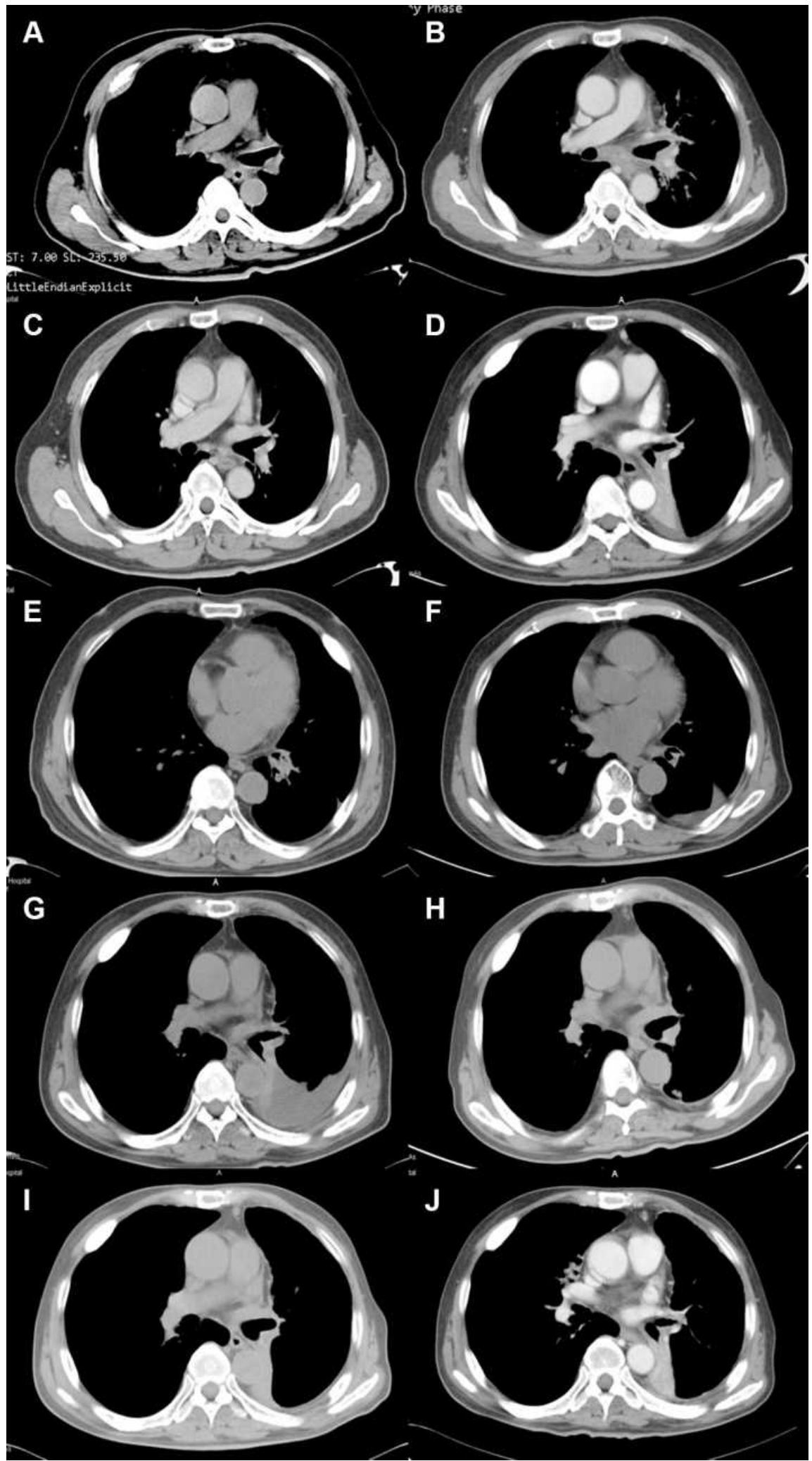

Figure 2 (A) Baseline CT Scan at diagnosis in Jan, 2013. (B) PD on chemotherapy in Aug, 2014. (C) PR after icotinib treatment in Feb, 2015. (D) PD on icotinib in Dec, 2015. (E) PR after osimertinib treatment in Sep, 2016. (F) PD on osimertinib in Dec, 2017. (G) PD on chemotherapy in Apr, 2018. (H) PR after the combined treatment of afatinib and apatinib in Nov, 2018. (I) PD on afatinib combined with apatinib treatment in Feb, 2019. (J) PR on albumin-bound paclitaxel and toripalimab treatment in Jul, 2019. 


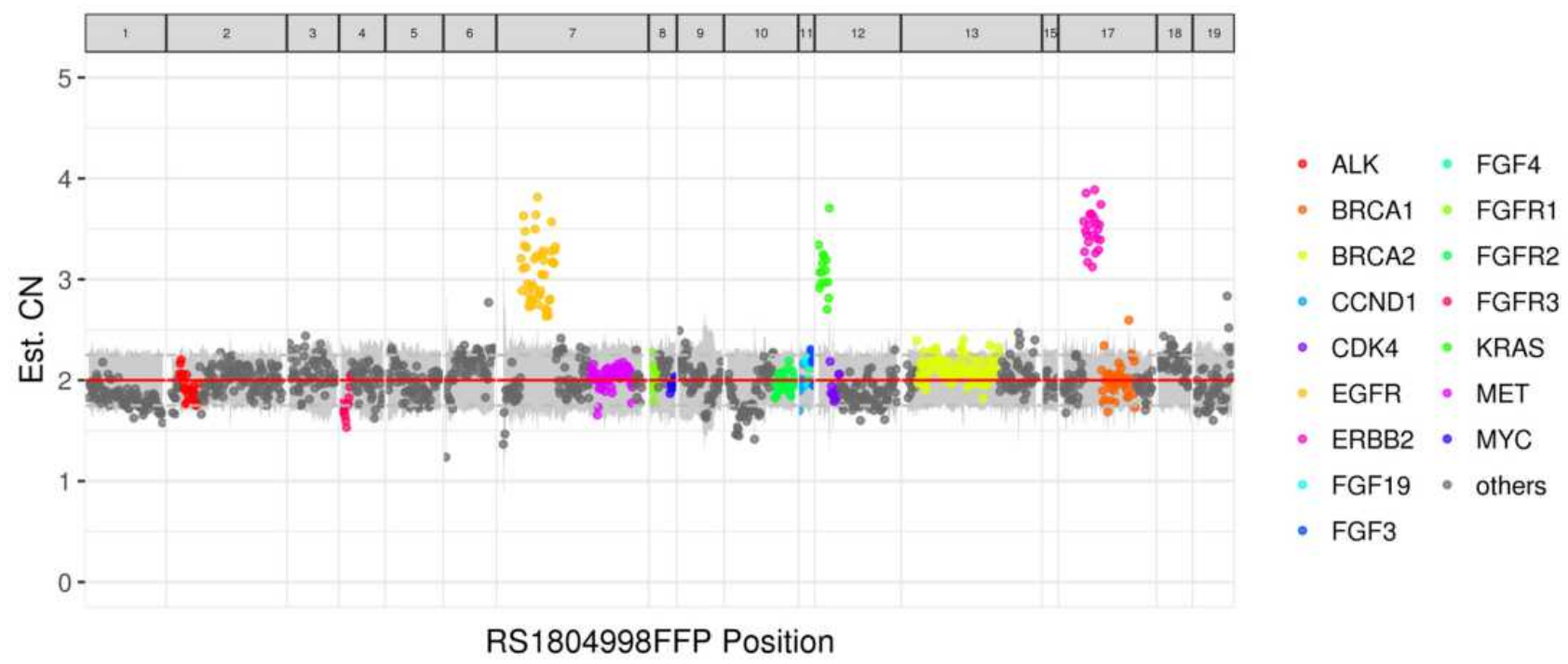

Figure 3 The distribution of gene copy number (purple blot: HER2 (ERBB2) amplification). Gene copy number variation (CNV) was identified based on the depth of coverage data of capture intervals. Because of GC content and target probe density, the coverage depth was corrected for the sequencing bias, and the average coverage of all capture regions was calculated as internal control. Gene amplification was defined when it met the following three criteria: I. Gene copy number is equal or greater than 2.75; 2 . The adjusted coverage depth of the gene in tumor samples is higher than internal control significantly (z-test). 3 . The coverage of the whole gene region is significantly higher than internal control ( $t$-test).

EGFR-TKIs alone as well as combined. ${ }^{3,4}$ Only some case reports showed that brigatinib combined with bevacizumab could be an effective treatment strategy and prolong the survival outcomes with NSCLC harboring EGFR C797S in cis with EGFR T790M..$^{5-7}$ On the other hand, it has been reported that $H E R 2$ amplification is identified in $2 \%$ of patients who have acquired resistance to secondline Osimertinib. ${ }^{8}$ In our case, we detect EGFR T790M-cis -C797S in plasma and HER2 amplification in tumor tissue that may both contribute to osimertinib resistance. The differences of NGS results between plasma and tumor tissue may attribute to the heterogeneity of tumors cells, which could be a key point in personalized medicine in treating patients with lung cancer. ${ }^{9}$

Afatinib, as an irreversible inhibitor for both EGFR and HER2, showed potent anti-tumor activity in HER2 amplified lung cancer cells in vitro and in vivo. ${ }^{10}$ A case report described a patient, who had developed HER2 amplification after gefitinib progression, showed partial response with afatinib treatment. ${ }^{11}$ Another patient with coexistent HER2 amplification and mutation received afatinib treatment and achieved PR with a PFS of 7 months. ${ }^{12}$ Although there were studies demonstrating afatinib had limited efficiency in treating HER2 aberrant NSCLC, ${ }^{13}$ afatinib was still a treatment option in heavily pretreated NSCLC patients with HER2 amplification. Apatinib, a small molecule TKI against VEGFR-2, was reported to prolong PFS when combined with EGFR-TKI in patients with acquired EGFR-TKI resistance. ${ }^{14}$ Moreover, a study demonstrated osimertinib combined with apatinib increased growth inhibition of lung cancer cells in vitro and in vivo, and three patients with $E G F R$ T790M lung adenocarcinomas responded to the combination therapy of osimertinib and apatinib after osimertinib single-agent failure, with PFS of 5-7 months. ${ }^{15}$ In our case, the combinatorial therapy of afatinib and apatinib was effective for pulmonary adenocarcinoma in the presence of EGFR C797S-cis-T790M and HER2 amplification after osimertinib failure and the disease remained stable for 10 months. However, therapeutic options for NSCLC patients with EGFR C797S-cis-T790M and $H E R 2$ amplification remain largely unknown and more research regarding the combination of afatinib and apatinib is needed.

\section{Conclusion}

In summary, our findings provide the first clinical evidence that afatinib plus apatinib may be a promising therapy for patients with EGFR C797S-T790M-19Del mutant and HER2 amplified NSCLC. After the failure of osimertinib treatment, dynamically monitoring through NGS provides important implications and possible opportunities for targeted therapy to overcome osimertinib resistance and prolong the survival of the patient. 


\section{Abbreviations}

EGFR, epidermal growth factor receptor; TKI, tyrosine kinase inhibitor; NSCLC, non-small cell lung cancer; PFS, progression-free survival; PR, partial response; OS, overall survival; $\mathrm{PD}$, progressive disease; $\mathrm{SD}$, stable disease; PD1, programmed cell death protein 1; HER2, receptor tyrosine-protein kinase erbB-2; VEGFR-2, vascular endothelial growth factor receptor 2; NMPA, National Medical Products Administration.

\section{Ethical Approval}

All procedures performed in studies involving human participants were in accordance with the Helsinki declaration. Institutional approval was not required to publish the case details.

\section{Patient Informed Consent}

The patient provided written informed consent to agree this case report for publication.

\section{Funding}

This work is funded by Zhenjiang Key Research and Development Program of China (grant NO. SH2018077) and $\mathrm{Wu}$ Jieping Medical Foundation (grant NO. 320.6750.19092-38).

\section{Disclosure}

Yuanyang Zhao and Yuxing Chen should be regarded as co-first authors. The authors declare no potential conflicts of interest.

\section{References}

1. Siegel RL, Miller KD, Jemal A. Cancer statistics, 2019. CA Cancer J Clin. 2019;69(1):7-34. doi:10.3322/caac.21551

2. Camidge DR, Pao W, Sequist LV. Acquired resistance to TKIs in solid tumours: learning from lung cancer. Nat Rev Clin Oncol. 2014;11 (8):473. doi:10.1038/nrclinonc.2014.104

3. Wang Z, Yang -J-J, Huang J, et al. Lung adenocarcinoma harboring EGFR T790M and in trans C797S responds to combination therapy of first-and third-generation EGFR TKIs and shifts allelic configuration at resistance. J Thorac Oncol. 2017;12(11):1723-1727. doi:10.1016/j. jtho.2017.06.017

OncoTargets and Therapy

\section{Publish your work in this journal}

OncoTargets and Therapy is an international, peer-reviewed, open access journal focusing on the pathological basis of all cancers, potential targets for therapy and treatment protocols employed to improve the management of cancer patients. The journal also focuses on the impact of management programs and new therapeutic
4. Arulananda S, Do H, Musafer A, Mitchell P, Dobrovic A, John T. Combination osimertinib and gefitinib in C797S and T790M EGFRmutated non-small cell lung cancer. $J$ Thorac Oncol. 2017;12 (11):1728-1732. doi:10.1016/j.jtho.2017.08.006

5. Zhao J, Zou M, Lv J, Han Y, Wang G, Wang G. Effective treatment of pulmonary adenocarcinoma harboring triple EGFR mutations of L858R, T790M, and cis-C797S by osimertinib, bevacizumab, and brigatinib combination therapy: a case report. Onco Targets Ther. 2018;11:5545. doi:10.2147/OTT.S170358

6. Wang X, Zhou L, Yin JC, Wu X, Shao YW, Gao B. Lung Adenocarcinoma harboring EGFR 19del/C797S/T790M triple mutations responds to brigatinib and Anti-EGFR antibody combination therapy. $J$ Thorac Oncol. 2019;14(5):e85-e88. doi:10.1016/j. jtho.2019.01.015

7. Wang Y, Yang N, Zhang Y, et al. Brief Report: effective treatment of lung adenocarcinoma harboring EGFR-activating mutation/T790M/ cis-C797S triple mutations by brigatinib and cetuximab combination therapy. J Thorac Oncol. 2020. doi:10.1016/j.jtho.2020.04.014

8. Mehlman C, Cadranel J, Rousseau-Bussac G, et al. Resistance mechanisms to osimertinib in EGFR-mutated advanced non-smallcell lung cancer: a multicentric retrospective French study. Lung Cancer. 2019;137:149-156. doi:10.1016/j.lungcan.2019.09.019

9. Lim Z-F, Ma PC. Emerging insights of tumor heterogeneity and drug resistance mechanisms in lung cancer targeted therapy. J Hematol Oncol. 2019;12(1):1-18. doi:10.1186/s13045-019-0818-2

10. Suzawa K, Toyooka S, Sakaguchi M, et al. Antitumor effect of afatinib, as a human epidermal growth factor receptor 2-targeted therapy, in lung cancers harboring HER 2 oncogene alterations. Cancer Sci. 2016;107(1):45-52. doi:10.1111/cas.12845

11. Meedendorp AD, Ter Elst A, Hart NA, Groen HJM, Schuuring E, van der Wekken AJ. Response to HER2 Inhibition in a patient with brain metastasis with EGFR TKI acquired resistance and an HER2 amplification. Front Oncol. 2018;8:176. doi:10.3389/ fonc. 2018.00176

12. Liu X, Cao Y, Li Y, Duan X. Advanced lung adenocarcinoma with coexistent HER2 mutation and amplification and response to afatinib: a case report. Ann Palliat Med. 2020;9(2):483-487. doi:10.21037/ apm.2020.02.14

13. Zhao J, Xia Y. Targeting HER2 alterations in non-small-cell lung cancer: a comprehensive review. JCO Precision Oncol. 2020;4 (4):411-425. doi:10.1200/PO.19.00333

14. Tian R, Song X, Guo Y, et al. P1. 14-42 Apatinib combined with EGFR-TKI in treating advanced non-small cell lung cancer with EGFR-TKI resistance (data updated). $J$ Thorac Oncol. 2019;14(10): S571. doi:10.1016/j.jtho.2019.08.1193

15. Liu Y, Xiong Z-C, Sun X, et al. Impact of apatinib in combination with osimertinib on EGFR T790M-positive lung adenocarcinoma. Transl Cancer Res. 2019;8(5):2151-2163. doi:10.21037/ tcr.2019.09.35

\section{Dovepress}

agents and protocols on patient perspectives such as quality of life, adherence and satisfaction. The manuscript management system is completely online and includes a very quick and fair peer-review system, which is all easy to use. Visit http://www.dovepress.com/ testimonials.php to read real quotes from published authors. 